\title{
Microvessel Density and Mammaglobin A Expression as Prognostic Markers in Molecular Types of Breast Cancer
}

\begin{abstract}
ADRIANA ANDREEA J ITARIU**, AMALIA RALUCA CEAUSU ${ }^{1}$, ADRIANA MECHE², CRISTIAN NICA ${ }^{3}$, AMELIA BURLEA², MARIUS RAICA ${ }^{1}$

IVictor Babes University of Medicine and Pharmacy, Department of Microscopic Morphology/Histology and Angiogenesis Research Center, 2 Eftimie Murgu Sq., 300041, Timisoara, Romania

${ }^{2}$ County Clinical Hospital, Department of Pathology, 2-4 Andreny Karoly Str., 310037, Arad, Romania

3Pius Brinzeu Emergency County Hospital, Department of General Surgery, 156 Liviu Rebreanu Blvd., 300723, Timisoara, Romania

Increased microvessel density (MVD) values in breast cancer correlate with tumor growth and progression while mammaglobin (MGB) expression in tumor cells is associated with a favorable prognosis. We aim to evaluate and correlate MVD values with MGB expression in molecular types of breast cancer specimens and to determine their utility as prognostic biological markers. A number of 52 breast cancer specimens were included in the study. Specimens were processed for routine histopathological diagnosis followed by the molecular classification by means of estrogen (ER), progesterone $(P R)$ and HER2 immunohistochemical reactions. After performing immunohistochemistry for CD34 and MGB, MVD evaluation was made using the "hot spot" method for each case and MGB was scored between 0 (negative) and +3 (strong positive) depending on the intensity and distribution of the staining. MGB expression in tumor cells and MVD mean values were extremely variable. The greatest MVD mean values were obtained in luminal $B$ followed by HER2, luminal $A$ and triple negative breastcancer (TNBC) (95.33, 69, 62, and 40, respectively). MGB expression in the tumor cells generally ranged from mild to weak and was strong only in a few invasive ductal carcinoma cases. In cases with TNBCs the expression of MGB in tumor cells was weak and focal or negative. This variability was noticed between the molecular types of breast cancers and even within the same molecular type. In a restricted number of cases, MGB positive tumors were associated with low MVD values while the negative cases were characterized by increased MVD mean values. The variable results we obtained regarding the correlation between MVD and MGB in breast cancer specimens may indicate a rather restricted use of MVD/MGB in estimating breast cancer patients' prognosis.
\end{abstract}

Keywords: breast cancer, angiogenesis, microvessel density, mammaglobin A, prognosis

MGB and MVD are known in the literature as prognostic parameters in breast cancer patients. Unlike MGB, MVD count is not restricted to the malignantly transformed breast tissue, its use being extended to a wide range of human benign and malignant conditions. MGB is one of the markers of the normal and malignantly transformed breast tissue where it can be detected in two main forms with different molecular masses [1, 2]. When investigating the human MGB gene, in 1998, Watson et al. [3] have concluded that it is not very well conserved phylogenetically and it presents a series of architectural similarities with uteroglobin genes and rat prostatein subunits. Using fluorescent in situ hybridization, the MGB gene has been localized on chromosome 11q13 which is usually amplified in breast cancer [3]. Increased MGB protein expression in breast cancer patients is associated with a favorable prognosis although its implications and functions are not yet fully understood [ 1,4$]$. MGB-A, one of the members of the secretoglobin superfamily, is expressed in mostbreastcancer cells and is currently being studied as a potential therapeutic target in immune therapies [5]. Its use in detecting both circulating and disseminated malignant cells explains MGB-A potential clinical applications [5]. Several studies have shown that MGB expression is strictly limited to the mammary tissue $[1,2]$ and that MGB-A represents a useful biomarker for detecting primary breast cancers but it also can be used to detect micrometastases in sentinel lymph nodes [6]. From this point of view, in a study conducted in 2010, Rehman et al. [7] have stated that MGB is overexpressed in breast carcinoma as compared to the normal breast epithelium' and that it 'can act as a useful tool in the diagnosis of women with breast cancer. Although being highly expressed in the early stages of breast cancer, MGB-A expression has been shown to decrease during the progression of this disease [8], thus pointing towards a poorer prognosis.

Although not being the focus of the study conducted by Sun etal., MGB has been shown to positively correlate with androgen receptor expression in invasive breast carcinomas [9]. In the histopathological types of breast cancer, MGB positivity has been demonstrated in mucinous carcinomas [10] and in cutaneous metastases from ductal breast carcinomas, its reaction being identified with an increased percentage [11]. Also, MGB-A expression is increased in typical and atypical intraductal papillomas of the breast but decreased in intraductal papillary carcinomas [12]. In case of the four molecular types of breast cancer, Lewis et al. have shown a variable MGB expression [13]. MGB expression is statistically significant in the luminal types and HER2 breast cancers whereas 'most BLCs [basal-like carcinomas] and UTNCs [unclassified triple-negative carcinomas] are negative for MGB [mammaglobin]' [13]. Despite its positive reaction in luminal-like and HER2 breast cancers, MGB is less sensitive than other markers [14].

High MGB expression levels are associated with decreased migration and invasion of breast cancer cells

*email: adriana_andreea_jitariu@yahoo.com; Phone: 0742/925280 
[2]. Several studies have shown that MGB-derived peptides are capable of inducing T-cell mediated immune responses [15], thus becoming potential targets in immunotherapy based breast cancer treatment. This hypothesis is based on the fact that some of the MGB-derived proteins are expressed on the surface of breast cancer cells and may consecutively be used for breast cancer targeted drug delivery [15, 16]. Prior to these studies, Guan et al. have demonstrated that MGB-A is not only a breast-specific protein but also an independent breast cancer prognostic marker due to the lack of correlation neither with progesterone receptors nor with the Nottingham grade [17]. Besides its diagnostic and prognostic role in breast cancers, MGB-A is also regarded as a useful differential marker of breast cancer metastasis from primary lung cancer [18].

Despite its exclusive expression in the mammary tissue, in the past, MGB has been insufficientlystudied in the field of breast cancer. Very few studies have been focused on demonstrating the role of this protein in the major histopathological types of breast cancer, and, even fewer, on presenting the implications of MGB-A in the molecular types of breast cancer. The available data regarding the implications of MGB-A in breast cancer either has notbeen or has been insufficiently correlated with MVD, an indicator of tumor angiogenesis in breast cancer specimens. Increased MVD values correlate with a poorer prognosis and with a higher metastatic risk in several human cancers, including those of the breast [19]. The correlation between MVD and different molecular substances, such as indoleamine 2,3-dioxygenase (IDO) in advanced stages of breast cancer has recently been demonstrated [20]. Using contrast-enhanced ultrasonography, Li X et al. have shown the importance of MVD not only as a prognostic parameter but also as an indirect indicator of the histological grade of breast cancers [21]. As an indicator of active angiogenesis in benign and malignant human lesions, MVD values are influenced by several growth factors, hormones [22] and even by tryptase positive mast cells [23, 24]. Furthermore, Liu et al. found a statistically significant association between microRNA(miR)-10b, a predictor of metastases and MVD values [25]. Downregulation of tumor vessel proliferation and the decrease of MVD values are one of the key points in antiangiogenic therapy and improvement of the overall survival rate [26]. A favorable patient prognosis was strongly associated with low MVD values and with a decrease of different matrix metalloproteinases [27], known to promote cancer cell migration. MVD measurement following CD31, CD34 or CD105 immunohistochemical assessment was associated with a high metastatic risk in patients diagnosed with breast cancer in case the values were increased [28, 29]. Due to their implications as prognostic biomarkers and indicators of patient overall survival rate, we propose to analyze the relationship between MGB-A expression in the tumor cells and MVD in the molecular types of breast cancer. MVD/MGB-A association may bring new insights on the understanding of breast cancer evolution and impact on the patients outcome.

\section{Experimental Part}

\section{Materials and methods}

A total number of 52 cases of breast carcinomas were included in our study. Tissue specimens were fixed in $10 \%$ buffered formalin for 48 hours and paraffin embedded. Five $\mu m$ thick sections were performed from each paraffin block and sections were mounted on silanized slides. Sections from each case were stained with routine hematoxylin and eosin method for histopathologic examination using Axiocam 506 color, Zeiss, J ena, Germany. Out of the total number of cases, 38 cases were ductal invasive carcinoma, 7 cases of invasive lobular carcinoma, 1 medullary carcinoma and 6 cases of hybrid tumors as follows: ductal in situ carcinoma and ductal invasive carcinoma, ductal invasive carcinoma and medullary carcinoma, ductal invasive and lobular invasive carcinoma, respectively. Out of the total number of 38 cases of ductal invasive carcinomas, 11 were associated with their lymph node metastases.

For the molecular classification of breast cancer, all the specimens were stained for ER, PR and HER2. Immunohistochemical techniques included heat-induced epitope retrieval with Bond Epitope Retrieval Solution 2, a ready-to-use, $\mathrm{pH} 9.0$ solution (Leica Biosystems, Newcastle Ltd, Newcastle Upon Tyne NE 12 8EW, UK) for 20 minutes, in the case of the ER and PR antibodies. Endogenous peroxidase blocking was performed using 3\% hydrogen peroxide for 5 minutes. ER (monoclonal, clone 6F11, Bond ready-to-use reagent, Leica Biosystems, Newcastle Upon Tyne, UK, 30 minutes incubation time) and PR (monoclonal, clone 16, Bond ready-to-use reagent, Leica Biosystems, Newcastle Upon Tyne, UK, 30 minutes incubation time) were used as primary antibodies. The Bond Polymer Refine Detection System was used for visualisation. 3, 3 diamino-benzidine dihydrochloride was applied for 10 minutes as chromogen, and haematoxylin for 5 minutes as nuclear staining. The Bond Oracle Detection System was used for HER2 visualisation. Immunoreactivity was estimated as positive in the cells with nuclear (ER, PR) and membrane expression (HER2). The entire immunohistochemical procedure was performed with Leica Bond-Max (Leica Biosystems, Newcastle Upon Tyne, UK) autostainer. Out of the total number of cases included in our study, 34 cases were classified as luminal A, 14 cases as luminal B, 2 cases as HER 2 and 2 cases as TNBCs.

The molecular classification was followed by MGB detection in tumor cells and tumor vessels count after using CD34. For the double immunostaining, CD34 (monoclonal, clone QBEnd10, Bond ready to use reagent, Leica Biosystems, Newcastle Upon Tyne, UK, 30 minutes incubation time) and MGB (monoclonal, clone 304-1A5, ready to use, DakoCytomation, California Inc., $30 \mathrm{~min}$ incubation time) were used as antibodies. The Bond Polymer Refine Detection System and Bond Polymer Refine Red Detection System were used for visualisation. Cytoplasmic expression (MGB - red, CD34 - brown) was evaluated for MVD calculation using the hot spot method by choosing the microscopic field containing the highest vessel density for each specimen under $X 4$ magnification. Vessel counting was performed under $X 20$ magnification by choosing 3 different areas from the same initially selected microscopic field. CD34 positive vessels (brown) were counted separately followed by the calculation of the arithmetic mean for each selected field. MGB positive tumor cells (red) were examined and characterized using a score that ranged from 0 (negative) to +3 (strong positive) depending on the distribution and staining intensity. Tumor cells were considered positive for MGB only if exhibiting a cytoplasmic pattern of reaction.

\section{Results and discussions}

All the specimens included in our study were characterized by the presence of CD34 positive vessels. Our results showed evident variability regarding MVD mean values and MGB expression in breast cancer tumor cells. 
This variability was found between the different molecular types of breast cancer and even between the same molecular types.

MGB cytoplasmic pattern of expression was mostly diffuse and homogeneous. In these specimens we noticed that MGB positive cells invaded the surrounding adipose tissue (fig. 1).

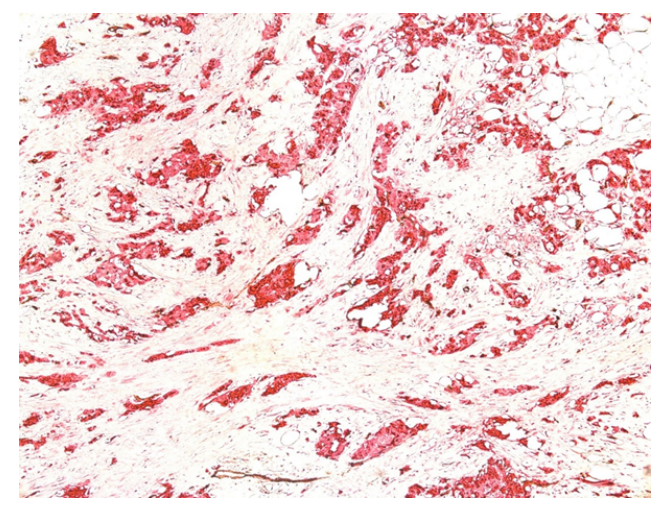

Fig. 1. Invasive ductal carcinoma showing positive MGB expression in the tumor cells. Note the low number of CD34 positive vessels (brown) and the presence of numerous MGB positive tumor cells (red) in the areas of white adipose tissue invasion (upper right).

Note the diffuse MGB expression in all tumor areas. X10 magnification.

The positive reaction for MGB was maintained in the areas of tumor infiltration. For few breast cancer specimens we noticed an inversely proportional correlation between MVD and MGB. In these cases, the strong MGB expression in the tumor cells was associated with low MVD mean values, whereas the negative reaction for MGB expression was associated with increased MVD.

Most of the vessels were of small caliber, with a thin vascular wall, either with a narrow or without visible lumen (fig. 2). These vessels were found in both peritumor and intratumor areas.

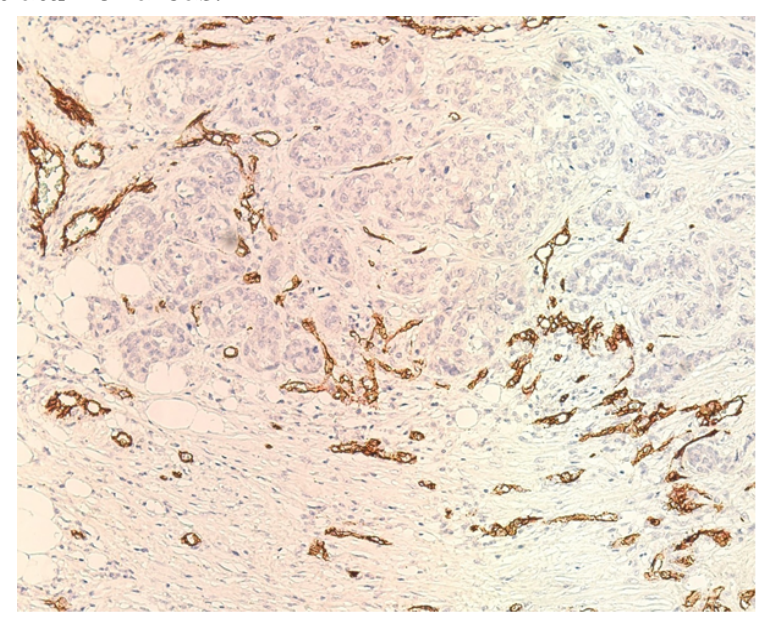

Fig.2. Invasive ductal carcinoma showing MGB negative tumor cells. Note the presence of numerous CD34 positive vessels of small caliber. X10 magnification.

In lymph node metastases, the vessels surrounding the tumor areas and those situated at a distance from these areas all present a CD34 positive immunohistochemical profile (fig 3). Most of the lymph node metastases exhibited an MGB negative profile. However, we did noticed a positive MGB reaction that ranged from strong to weak or was isolate in a few number of cases. The MGB immunohistochemical profile was generally maintained starting from the primary tumor to its lymph node metastases or it decreased from strong to weak. Despite these observations, we found one case of invasive ductal carcinoma luminal $B$ type where the corresponding lymph node metastasis was strongly positive for MGB (fig. 3).

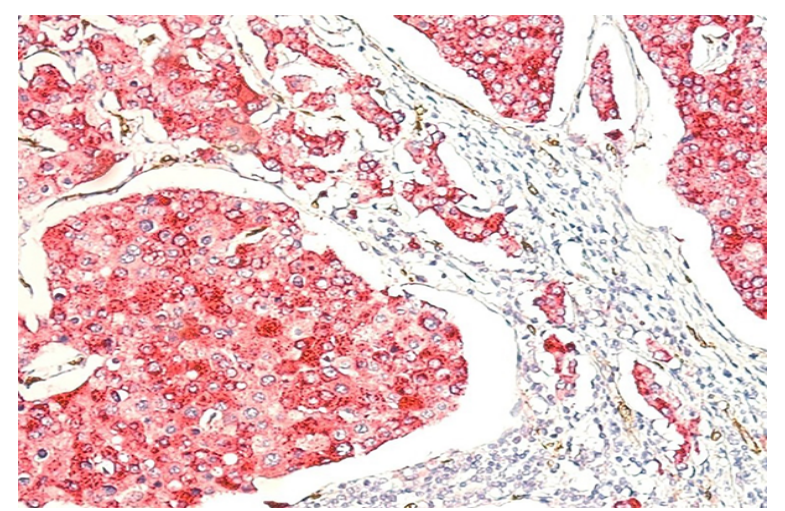

Fig. 3. Lymph node metastasis from an invasive ductal carcinoma exhibiting a solid growth pattern. Note the presence of small caliber vessels surrounding the tumor areas (brown) and the strong positive reaction for MGB in the tumor cells (red). MGB expression in the tumor cells is strong, diffuse and homogeneous, with a cytoplasmic pattern of reaction. X20 magnification.

The positive reaction for MGB in the tumor cells commences during the benign lesion stages and is weak in the intraductal hyperplasia areas. The intensity of MGB positive reaction gradually increases towards the atypical hyperplastic lesions. In the areas containing ductal carcinoma in situ, we found an MGB positive expression that was identical with the one exhibited by the invasive lesions.

All vessels were positive for $\mathrm{CD} 34$ but the intensity of the MGB reaction was extremely variable from one specimen to another and even within the same specimen (table 1). Some of the examined specimens showed a weak, focally positive MGB reaction.

The highest MVD mean value was obtained for luminal $B$ breast cancers (95.33) while the lowest value was obtained for the luminal A type (30). The mean value for TNBCs ranged between 62.66 and 40. MGB expression in the tumor cells was also variable but predominantly weak to mild with the exception of a few cases of invasive ductal carcinomas classiffied as luminal A and HER2 that were strong positive. In TNBCs cases MGB positive reaction was weak and focally distributed or was negative. Similarly to the other molecular types of breast cancer, TNBCs showed the presence of an increased MVD mean values. We noticed that the overall mean values for MVD and MGB expression in the tumor cells are variable from one breast cancer type to another and even between the same molecular types.

Despite being a promising prognostic biomarker, MGB values in the molecular types of breast cancer are controversial and insufficiently correlated with MVD in terms of diagnostic and prognostic applicability. From this point of view, GATA-3 possesses a higher diagnostic sensibility in invasive breast cancers and in cytologic materials [30, 31]. The highest GATA-3 expression was found in the luminal types of breast cancer [30]. However, GATA-3 utility in the diagnosis of poorly differentiated invasive breast cancers and non-luminal types is limited whereas MGB may become a useful diagnostic tool in these cases [30]. MVD, the major angiogenesis-related parameter [32] does not always correlate with MGB expression hence the difficulty of ensuring a complete estimation of the patients' prognosis. However, Hu et al. 


\begin{tabular}{|c|c|c|c|c|c|c|c|}
\hline \multicolumn{2}{|c|}{ Luminal A } & \multicolumn{2}{c|}{ Luminal } & \multicolumn{2}{c|}{ HER2 } & \multicolumn{2}{c|}{ TNBC } \\
\hline MVD & MGB & MVD & MGB & MVD & MGB & MVD & MGB \\
\hline 62 & +1 & 95.33 & +1 & 69 & +2 & 62.66 & +1 \\
\hline 38.66 & 0 & 59.33 & +1 & 48.66 & +3 & 40 & 0 \\
\hline 30 & +2 & & & 40.33 & 0 & & \\
\hline
\end{tabular}

Table 1

MVD MEAN VALUES AND THE CORRESPONDING MGB EXPRESSION IN THE MOLECULAR TYPES OF BREAST CANCER.

Legend: $\mathrm{MVD}=$ microvessel density; $\mathrm{MGB}=$ mammaglobin $\mathrm{A} ; \mathrm{TNBC}=$ triple negative

[33] have demonstrated the implications of MGB-A as an independent prognostic biomarker in breast cancer. Plasma MGB-A is associated with lymph node metastases and advanced tumor stage, but no correlation was found between MGB-A and tumor size, tumor differentiation, menopausal status and hormone status of breast cancer patients [33]. Our results show a variable MGB expression in the molecular types of breast cancer. Despite the general decrease of MGB expression starting from benign lesions to malignant changes of breast tissue specimens, a strong MGB expression in tumor cells is sometimes observed in invasive breast lesions. The relationship found by us between MVD and MGB expression in breast cancers is characterized by an increased variability. Thus, MGB positive specimens are not necessarily associated with low MVD values. Our findings suggest the possibility of an inversely proportional relation between MVD and MGB in a limited number of breast cancer specimens. The cases in which we identified a strong MGB expression and increased MVD values, may partially support the findings of Picot et al. regarding MGB-A implications in promoting cancer cell malignant features [34]. The authors of this study demonstrated that the loss of MGB-A expression determines a decrease of tumor cell proliferation [34]. However, these results need to be further investigated in order to become applicable in daily clinical practice.

Breast cancer angiogenesis is known to correlate with tumor progression and metastasis $[35,36]$ and thus with an aggressive profile and invasion potential. However, several previous studies have pinpointed the correlation between MGB and a favorable prognosis $[2,6]$. Our findings indicate that some invasive breast lesions maintain an intense MGB-A expression in the tumor cells, thus generating a series of questions in need of elucidation. Is MGB-A a friend or a foe for breast cancer patients? Could metastatic breast carcinomas that are MGB-A positive be defined as distinct molecular subtypes? Our study does not provide the only controversial results in the field of breast cancer research. In 2015, Kraby et al. [37] demonstrated that luminal A breastcancer was associated with a poor prognosis in the presence of a high MVD while basal-like breast cancers were not. In our study, the highest overall MGB and MVD mean values were obtained for luminal $A$ and luminal $B$ types followed by HER2 and TNBC (table 1). With reference to lymph node metastases, Raica et al. [38] have shown a significant correlation between MGB expression in the primary tumor and lymph node status but not with the pathologic subtype of breast carcinoma. These findings suggest that MGB expression only defines a subgroup of patients and is useful in order to detect breast cancer metastases [38]. These aspects support or results according to which combined MVD/MGB evaluation has a restricted use in estimating breast cancer patient's prognosis.

The results we obtained are variable between different molecular types of breast cancer and even between the same molecular types, thus suggesting the heterogeneous behavior pattern of breast cancer. Currently, MVD is documented as an independent prognostic factor for HER2 and negative phenotype breast cancers [39]. Increased MVD values are directly correlated with a poor survival rate $[37,39]$ especially for the negative profile types [39]. In accordance to these previous findings, the TNBCs specimens included in our study were negative for MGB, with the exception of a restricted area containing few MGB positive tumor cells that was detected in one of the two TNBCs cases. In these specimens MVD mean values were increased. It appears that the rate of angiogenesis is high in HER2 and TNBC compared to other breast cancers [40]. Our results support this finding through the high MVD mean values obtained for HER2 and TNBC cases. Recent studies show the importance of MVP (microvessel proliferation) in estimating the aggressive features of breast cancers [41] in comparison to standard MVD calculation. Although we only determined MVD and not MVP values for each case included in our study, all tumor specimens exhibited a rich vasculature.

MGB-A implications as a prognostic parameter in breast cancer patients remain controversial at the time being. The studies conducted in the field of breast cancer either support its negligible role compared to other biomarkers $[30,42]$ or are in favor of MGB-A implications in cancer cell proliferation and poor patient outcome [34]. Despite the available data, MGB may not be as completely elucidated as it seems at the time being. As far as we know, there are no current studies that have focused on the relation between MVD and MGB-A for the prognostic evaluation of the molecular types of breast cancer. The association between MGB-A and other prognostic parameters besides MVD may aid the emergence of novel and efficient biomarkers in the field of breast cancer diagnosis and prognosis.

\section{Conclusions}

Our results show no correlation between MVD and MGB in the molecular types of breast cancer. The use of combined MVD/MGB-A evaluation in estimating breast cancer patients' prognosis is restricted. With the exception of some few breast cancer specimens where we found an inversely proportional correlation between MVD mean values and MGB expression, most of the breast cancers included in our study exhibited a significant variability regarding both MVD mean values and MGB expression in the tumor cells. This variability was maintained in both the primary tumors and their associated lymph node metastases and in the different molecular profiles of breast cancer specimens. MGB expression was strong but mostly isolate in specimens containing lymph node metastasis. Some of the lymph node metastases were negative for MGB while others maintained a strong, diffuse MGB 
expression. Further studies in the field of breast cancer research are needed in order to confirm these findings.

Acknowledgements. The authors are grateful to Dr. Amelia Burlea and Dr. Adriana Meche for providing the tissue specimens and to Patricia Berzava and Ciprian Oniga for their excellenttechnical support. This work has been supported by the Internal Competition Program, Projects for Young Researchers PII-C5-TC-2017-01, granted by the Victor Babes University of Medicine and Pharmacy Timi'oara, Romania and with kind support from the County Clinical Hospital Arad, Romania.

\section{References}

1.MARGAN, M., M., JITARIU, A., A., CIMPEAN, A., M., NICA, C., RAICA, M., Molecular Portrait of the Normal Human Breast Tissue and Its Influence on Breast Carcinogenesis, J. Beast Cancer., 19, nr. 2, 2016, p. 99-111.

2.KOH, E., H., CHO, Y., W., MUN, Y., J., RYU, J., H., KIM, E., J., CHOI, D., S., MAENG, K., Y., HAN, J., KANG, D., Upregulation of human mammaglobin reduces migration and invasion of breast cancer cells, Cancer Invest., 32, nr. 1, 2014, p. 22-29.

3.WATSON, M., A., DARROW, C., ZIMONJIC, D., B., POPESCU, N., C., FLEMING, T., P., Structure and transcriptional regulation of the human mammaglobin gene, a breast cancer associated member of the uteroglobin gene family localized to chromosome 1lq13, Oncogene., 16, nr. 6, 1998, p. 817-824.

4.HUANG, Y., ZHANG, H., Q., WANG, J., SONG, X., G., WANG, G., $H_{\text {., }}$ GUAN, Q., ZHO, W., HUO, F., YU, C., Z., ZENG, Y., J., Cloning expression, monoclonal antibody preparation and serologic study of mammaglobin in breast cancer, Neoplasma., 58, nr. 5, 2011, p. 436440.

5.GHERSEVICH, S., CABELLOS, M., P., Mammaglobin A: review and clinical utility, Adv. Clin. Chem., 64, 2014, p. 241-268.

6.TAFRESHI, N., K., ENKERMANN, S., A., BUI, M., M., LLOYD, M., C., ABRAHAMS, D., HUYNH, A., S., et al., A mammaglobin-A targeting agent for noninvasive detection of breast cancer metastasis in lymph nodes, Cancer Res., 71, nr. 3, 2011, p. 1050-1059.

7.REHMAN, F., NAGI, A., H., HUSSAIN, M., Immunohistochemical expression and correlation of mammaglobin with the grading system of breast carcinoma, Indian J. Pathol. Microbiol., 53, nr. 4, 2010, p. 619623.

8.BOZHENKO, V., K., KHARCHENKO, N., V., VASKEVICH, E., F., KUDINOVA, E., A., OORZHAK, A., V., ROZHKOVA, N., I., TROTSENKO, I., D., Mammaglobin in peripheral blood and tumor breast cancer patients, Biomed Khim., 62, nr. 4, 2016, p. 453-457.

9.SUN, X., J., ZUO, K., TANG, S., X., LU, H., F., SHUI, R., H., YU, B., H., $X U, W ., L_{.}$, CHENG, Y., F., TU, X., Y., BI, R., YANG, W., T., Correlation between androgen receptor expression and surrogate molecular subtypes in invasive breast carcinoma, Zhonghua Bing Li Xue Za Zhi., 46, nr. 7, 2017, p. 476-480.

10.DE ANDRADE NATAL, R., DERCHAIN, S., E., PAVANELLO, M., PAIVA, G., R., SARIAN, L., O., VASSALLO, J., Expression of unusual immunohistochemical markers in mucinous breast carcinoma, Acta Histochem., 119, nr. 3, 2017, p. 327-336.

11.FERNANDEZ-FLORES, A., Immunohistochemical and morphologic evaluation of primary cutaneous apocrine carcinomas and cutaneous metastases from ductal breast carcinoma, Rom. J. Morphol. Embryol., 53, nr. 4, 2012, p. 879-892.

12.ZHANG, H., XIONG, Y., ZHANG, S., ZHANG, Y., WANG, Y., H., LI, T., Clinicopathologic and immunohistochemical study of 187 cases of intraductal papillary neoplasm of breast, Zhonghua Bing Li Xue Za Zhi., 40, nr. 11, 2011, p. 726-731.

13.LEWIS, G., H., SUBHAW ONG, A., P., NASSAR, H., VANG, R., ILLEI, P., B., PARK, B., H., ARGANI, P., Relationship between molecular subtype of invasive breast carcinoma and expression of gross cystic disease fluid protein 15 and mammaglobin, Am. J. Clin. Pathol., 135, nr. 4, 2011, p. 587-591.
14.SHAOXIAN, T., BAOHUA, Y., XIAOLI, X., YUFAN, C., XIAOYU, T., HONGFEN, L., RUI, B., XIANGJIE, S., RUOHONG, S., WENTAO, Y., Characterisation of GATA3 expression in invasive breast cancer: differences in histological subtypes and immunohistochemically defined molecular subtypes, J. Clin. Pathol., 70, nr. 11, 2017, p. 926934.

15.GOEDEGEBUURE, P., S., WATSON, M., A., VIEHL, C., T., FLEMING, T., P., Mammaglobin-based strategies for treatment of breast cancer. Curr Cancer Drug Targets, 4, nr. 6, 2004, p. 531-542.

16.ZUO, L., LI, L., WANG, Q., FLEMING, T., P., YOU, S., Mammaglobin as a potential molecular target for breast cancer drug delivery, Cancer Cell Int., 9, 2009, p. 8.

17.GUAN, X., F., HAMEDANI, M., K., ADEYINKA, A., WALKER, C., KEMP, A., MURPHY, L., C., WATSON, P., H., LEYGUE, E., Relationship between mammaglobin expression and estrogen receptor status in breast tumors, Endocrine., 21, nr. 3, 2003, p. 245-250.

18.SASAKI, E., TSUNODA, N., HATANAKA, Y., MORI, N., IWATA, H., YATABE, Y., Breast-specific expression of MGB1/mammaglobin: an examination of 480 tumors from various organs and clinicopathological analysis of MGB1-positive breast cancers, Mod. Pathol., 20, nr. 2, 2007, p. 208-214.

19.ZHANG, J., ZHANG, L., LIN, Q., REN, W., XU, G., Prognostic value of endoglin-assessed microvessel density in cancer patients: a systemic review and meta-analysis, Oncotarget., 9, nr. 7, 2017, p. 7660-7671.

20.WEI, L., ZHU, S., LI, M., LI, F., WEI, F., LIU, J., REN, X., High Indoleamine 2,3-Dioxygenase Is Correlated With Microvessel Density and Worse Prognosis in Breast Cancer, Front Immunol., 9, 2018, p. 724.

21.LI, X., LI, Y., ZHU, Y., FU, L., LIU, P., Association between enhancement patterns and parameters of contrast-enhanced ultrasound and microvessel distribution in breast cancer, Oncol. Lett., 15, nr. 4, 2018, p. 5643-5649.

22.BALINISTEANU, B., CIMPEAN, A., M., MELNIC, E., COCULESCU, M., CEAUSU, R., A., RAICA, M., Crosstalk between tumor blood vessels heterogeneity and hormonal profile of pituitary adenomas: evidence and controversies, Anticancer Res., 34, nr. 10, 2014, p. 5413-5420.

23.MARECH, I., AMMENDOLA, M., LEPORINI, C., PATRUNO, R., LUPOSELLA, M., ZIZZO, N., PASSANTINO, G., SACCO, R., FAROOQI, A., A., ZUCCALA, V., LEO, S., DENTAMARO, R., PORCELLI, M., GADALETA, P., DE SARRO, G., GADALETA, C., D., RANIERI, G., C-Kit receptor and tryptase expressing mast cells correlate with angiogenesis in breast cancer patients, Oncotarget., 9, nr. 8, 2017, p. 7918-7927.

24.RAICA, M., CIMPEAN, A., M., RIBATTI, D., Angiogenesis in premalignant conditions, Eur. J. Cancer., 45, nr. 11, 2009, p. 1924-1934.

25.LIU, X., GUAN, Y., WANG, L., NIU, Y., MicroRNA-10b expression in node-negative breast cancer-correlation with metastasis and angiogenesis, Oncol. Lett., 14, nr. 5, 2017, p. 5845-5852.

26.PIRTEA, L., RAICA, M., CIMPEAN, A., M., Endothelial cell activation and proliferation in ovarian tumors: two distinct steps as potential markers for antiangiogenic therapy response, Mol. Med. Rep., 5, nr. 5, 2012, p. 1181-1184.

27.XU, T., ZHANG, L., XU, H., KANG, S., XU, Y., LUO, X., HUA, T., TANG, G., Prediction of low-risk breast cancer using quantitative DCEMRI and its pathological basis, Oncotarget., 8, nr. 69, 2017, p. 114360114370.

28.SUN, C., LI, J., WANG, B., SHANGGUAN, J., FIGINI, M., SHANG, N., PAN, L., ZHANG, Z., Tumor angiogenesis and bone metastasis Correlation in invasive breast carcinoma, J. Immunol. Methods., 452, 2018, p. 46-52.

29.GUO, J., L., GU, S., Q., LI, Y., ZHANG, X., Y., Evaluation of clinical significance of endoglin expression during breast cancer and its correlation with ER and PCNA, Eur. Rev. Med. Pharmacol. Sci., 21, nr. 23, 2017, p. 5402-5407.

30.NI, Y., B., TSANG, J., Y., S., SHAO, M., M., CHAN, S., K., CHEUNG, S., Y., TONG, J., TO, K., F., TSE, G., M., GATA-3 is superior to GCDFP-15 and mammaglobin to identify primary and metastatic breast cancer, Breast Cancer Res. Treat., 169, nr. 1, 2018, p. 25-32. 
31.HAFEZ, N., H., SHAABAN, H., M., Can GATA-3 Immunohistochemistry be Utilized as a Reliable Diagnostic Marker for Metastatic Breast Carcinoma in Cytological Materials? A Comparative Study with Mammaglobin and GCDFP-15 Expression, Turk Patoloji Derg, 13, 2017, p. 1-7.

32.SHRIVASTAV, S., BAL, A., SINGH, G., JOSHI, K., Tumor Angiogenesis in Breast Cancer: Pericytes and Maturation Does Not Correlate With Lymph Node Metastasis and Molecular Subtypes, Clin. Breast Cancer., 16, nr. 2, 2016, p. 131-138.

33.HU, Y., LIU, P., WU, D., JIANG, Y., Prognostic role of plasma mammaglobin $A$ expression in breast carcinoma patients: a metaanalysis, Onco. Targets Ther., 11, 2018, p. 3245-3255.

34.PICOT, N., GUERRETTE, R., BEAUREGARD, A., P., JEAN, S., MICHAUD, P., HARQUAIL, J., Mammaglobin 1 promotes breast cancer malignancy and confers sensitivity to anticancer drugs, Mol. Carcinog., 55, nr. 7, 2016, p. 1150-1162.

35.ZHOU, Z., YAO, H., HU, H., Disrupting Tumor Angiogenesis and The Hunger Games for Breast Cancer, Adv. Exp. Med. Mol., 1026, 2017, p. 171-195.

36.KOPEC, M., ABRAMCZYK, H., Angiogenesis -a crucial step in breast cancer growth, progression and dissemination by Raman imaging, Spectrochim. Acta A. Mol. Biomol. Spectrosc., S1386-1425, nr. 18, 2018, p. 30175-30176.
37.KRABY, M., R., KRUGER, K., OPDAHL, S., VATTEN, L., J., AKSLEN, L., A., BOFIN, A., M., Microvascular proliferation in luminal $A$ and basal-like breast cancer subtypes, J. Clin. Pathol., 68, nr. 11, 2015, p. 891-897.

38.RAICA, M., CIMPEAN, A., M., MECHE, A., ALEXA, A., SUCIU, C., MURESAN, A., Analysis of the immunohistochemical expression of mammaglobin A in primary breast carcinoma and lymph node metastasis, Rom. J. Morphol. Embryol., 50, nr. 3, 2009, p. 341-347.

39.KRABY, M., R., OPDAHL, S., AKSLEN, L., A., BOFIN, A., M., Quantifying tumor vascularity in non-luminal breast cancers, J. Clin. Pathol., 70, nr. 9, 2017, p. 766-774.

40.KEYHANI, E., MUHAMMADNEJAD, A., BEHJ ATI, F., SIRATI, F., KHODADADI, F., KARIMLOU, M., MOGHADDAM, F., A., PAZHOOMAND, $\mathrm{R}$., Angiogenesis markers in breast cancer-potentially useful tools for priority setting of angiogenic agents, Asian Pac. J. Cancer Prev., 14, nr. 12, 2013, p. 7651-7656.

41.BUJ OR, I., S., CIOCA, A., CEAUSU, R., A., VEACESLAV, F., NICA, C., CIMPEAN, A., M., RAICA, M., Evaluation of Vascular Proliferation in Molecular Subtypes of Breast Cancer, In Vivo., 32, nr. 1, 2018, p. 79-83. 42.NI, Y., B., TSANG, J., Y., CHAN, S., K., TSE, G., M., GATA-binding protein 3 , gross cystic disease fluid protein-15 and mammaglobin have distinct prognostic implications in different invasive breast carcinoma subgroups, Histopathology., 67, nr. 1, 2015, p. 96-105.

$\overline{\text { Manuscript received: } 19.10 .2018}$ 